\title{
APPLICATIONS OF AMENABLE SEMIGROUPS IN OPERATOR THEORY
}

\author{
PIOTR NIEMIEC AND PAWEŁ WÓJCIK
}

\begin{abstract}
The paper deals with continuous homomorphisms $\mathscr{S} \ni s \mapsto T_{s} \in$ $\mathscr{L}(E)$ of amenable semigroups $\mathscr{S}$ into the algebra $\mathscr{L}(E)$ of all bounded linear operators on a Banach space $E$. For a closed linear subspace $F$ of $E$, sufficient conditions are given under which there exists a projection $P \in \mathscr{L}(E)$ onto $F$ that commutes with all $T_{s}$. And when $E$ is a Hilbert space, sufficient conditions are given for the existence of an invertible operator $L \in \mathscr{L}(E)$ such that all $L T_{s} L^{-1}$ are isometries. Also certain results on extending intertwining operators, renorming as well as on operators on hereditarily indecomposable Banach spaces are offered.
\end{abstract}

\section{INTRODUCTION}

A (semi)group $\mathscr{S}$ is amenable if there exists a (so-called) invariant mean on a suitably chosen vector space of (certain) bounded real-valued functions defined on $\mathscr{S}$. This mean is a positive linear functional invariant under (left, right or both left and right) translations of the semigroup. The so-called Banach limits on $\ell_{\infty}$ (see e.g. II.4.22 on page 73 in 10) are the most classical examples of invariant means on a semigroup that is not a group. The above (sketch of a) definition of an amenable semigroup deals with an intrinsic property of the semigroup. However, the amenability is equivalent to a strong (and powerful) property of a fixed point. Namely, a semigroup $\mathscr{S}$ is amenable iff for every affine action (with certain additional properties related to continuity and corresponding to the version of amenability we deal with) $K \ni x \mapsto \phi_{s}(x) \in K(s \in \mathscr{S})$ of $\mathscr{S}$ on a compact convex non-empty subset $K$ of a locally convex topological vector space there is a point $a \in K$ such that $\phi_{s}(a)=a$ for all $s \in \mathscr{S}$. This property makes amenability strongly applicable and thus amenable structures (which include locally compact topological groups, abstract semigroups, extremely amenable Polish groups, Banach algebras, $C^{*}$-algebras) are still widely investigated and prospected. The simplest criterion for a topological group (or a semigroup) to be amenable is the commutativity of its binary action. However, there are a number of amenable groups (in particular, all compact topological) that are non-abelian. The reader interested in the classical notion of amenability (for topological groups or semigroups) is referred to, e.g., 7], [17, 18, or Chapter 1 in 22.

In this paper we propose two versions of amenability (see Definition 3.1 in Section 3 below). Because of applications offered in this paper, we focus only on right amenability. Our considerations comprise all semigroups equipped with arbitrary

2010 Mathematics Subject Classification. Primary 47D03; Secondary 43A07, 47B40, 47A15, 47H10, 46B28.

Key words and phrases. bounded semigroup of operators; power bounded operator; decomposition of an operator; similarity to an isometry; amenable semigroup; abelian semigroup; fixed point property.

The first author is supported by the NCN (National Science Center in Poland), decision No. DEC-2013/11/B/ST1/03613. 
topologies, that form a much wider class than topological semigroups. This is motivated by a growing interest in semitopological semigroups (or Ellis semigroups; these are related to ultrafilter techniques) in dynamical systems and ergodic theory (see, e.g., Chapter 6 in [1]). We offer here certain results on: the existence of projections commuting with a collection of bounded operators on a Banach space (Propositions 4.2 and 4.5. Theorems 4.6 and 4.7 and Corollary 4.8); extending intertwining operators (Theorem 4.10 and Corollary 4.11); renorming the space in a way that all operators from a given family become isometric (Theorem 4.13 and Corollary 4.14); as well as joint similarity to isometries in a Hilbert space (Proposition 4.16 and Corollary 4.17). For clarity and simplicity, below we formulate main of these results in the case of abelian familes of operators:

1.1. Theorem. Let $\mathscr{T} \subset \mathscr{L}(E)$ be an abelian multiplicative semigroup of operators on a Banach space $E$, and $F$ be a closed linear subspace of $E$ such that:

(1) for any $T \in \mathscr{T}, T(F)=F$ and the restriction $T \uparrow F$ of $T$ to $F$ is an isomorphism;

(2) $F$ is a dual Banach space; that is, $F$ is linearly isometric to the dual space of some Banach space (and thus the closed unit ball $B_{F}$ of $F$ is compact with respect to certain locally convex topology, called further weak*, of $F$ );

(3) for any $T \in \mathscr{T}, T \uparrow F$ is weak* continuous on $B_{F}$;

(4) $\sup _{T \in \mathscr{T}}\left(\|T\| \cdot\left\|(T \uparrow F)^{-1}\right\|\right)<\infty$.

Then there exists a projection $P \in \mathscr{L}(E)$ onto $F$ that commutes with all $T \in \mathscr{T}$ iff $F$ is complemented in $E$. More specifically, if $F$ is complemented in $E$, then there exists a projection $Q \in \mathscr{L}(E)$ onto $F$ that commutes with all operators in $\mathscr{T}$ and satisfies:

- $\|Q\| \leqslant \sup _{T \in \mathscr{T}}\left(\|T\| \cdot\left\|(T \uparrow F)^{-1}\right\|\right) \cdot \lambda(E, F)$, where $\lambda(E, F)=\inf \{\|P\|: P \in$ $\mathscr{L}(E)$ a projection onto $F$ \},

- $Q$ is minimal; that is, $\|Q\| \leqslant\|P\|$ for any other projection $P$ onto $F$ that commutes with all operators in $\mathscr{T}$.

A special case (for compact groups) of the generalisation of the above result (formulated in Proposition 4.2) is due to Rudin [20].

1.2. Theorem. Let $\mathscr{S} \ni s \mapsto A_{s} \in \mathscr{L}(X)$ and $\mathscr{S} \ni s \mapsto B_{s} \in \mathscr{L}(Y)$ be two homomorhisms, with $B_{s}$ invertible for all $s \in \mathscr{S}$, of an abelian semigroup $\mathscr{S}$, where $X$ is a Banach space and $Y$ is a dual Banach space. Further, let $T_{0}: E \rightarrow Y$ be a bounded linear operator from a closed linear subspace $E$ of $X$ into $Y$ such that for any $s \in \mathscr{S}, A_{s}(E) \subset E$ and $B_{s} T_{0}=T_{0} A_{s} \uparrow E$. Then the following conditions are equivalent:

(i) $T_{0}$ extends to a bounded linear operator $T: X \rightarrow Y$ such that $B_{s} T=T A_{s}$ for all $s \in \mathscr{S}$;

(ii) there exists a bounded linear operator $T^{\prime}: X \rightarrow Y$ that extends $T_{0}$ and satisfies $\sup _{s \in \mathscr{S}}\left\|B_{s}^{-1} T^{\prime} A_{s}\right\|<\infty$.

1.3. Theorem. Let $\mathscr{T} \subset \mathscr{L}(E)$ be a bounded abelian multiplicative semigroup of operators on a Banach space $E$, and $\mathcal{N}(\mathscr{T}) \stackrel{\text { def }}{=}\{x \in E: T x=x(T \in \mathscr{T})\}$ and $\mathcal{R}(\mathscr{T}) \stackrel{\text { def }}{=} \varlimsup \operatorname{lin}\{T x-x: T \in \mathscr{T}, x \in E\}$. Then:

- $\mathcal{N}(\mathscr{T}) \cap \mathcal{R}(\mathscr{T})=\{0\}$,

- the subspace

$$
\mathcal{D}(\mathscr{T}) \stackrel{\text { def }}{=} \mathcal{N}(\mathscr{T})+\mathcal{R}(\mathscr{T})
$$

is closed in E, and

- the projection $P: \mathcal{D}(\mathscr{T}) \rightarrow \mathcal{N}(\mathscr{T})$ induced by the decomposition (1-1) has norm not greater than $\sup _{T \in \mathscr{T}}\|T\|$. 
1.4. Theorem. Let $\mathscr{T} \subset \mathscr{L}(H)$ be a bounded abelian multiplicative semigroup of operators on a Hilbert space $H$. Suppose there are two positive real constants $m$ and $M$ such that for any $T \in \mathscr{T}$ and $x \in H$,

$$
m\|x\| \leqslant\|T x\| \leqslant M\|x\| .
$$

Then there exists an invertible positive operator $A \in \mathscr{L}(H)$ such that $m I \leqslant A \leqslant M I$ and $A T A^{-1}$ is an isometry for each $T \in \mathscr{T}$.

The above theorem generalises a classical result of Sz.-Nagy [24] on bounded abelian groups of Hilbert space operators.

The paper is organised as follows. In the next section we introduce basic notions and fix the notation. Section 3 is a preliminary part on two variants of amenability we will deal with further. The fourth, main part is devoted to applications of amenablility defined in the previous one. We prove and formulate there all results dealing with amenable semigroups and operators in Banach spaces. This section is concluded by a proof of Theorems 1.1 1.3. In the last, fifth section we give more information on the two kinds of amenability introduced in Section 3.

\section{Notation And TERminology}

In this paper all semigroups are non-empty and Banach spaces are real or complex. We use multiplicative notation to denote binary actions of abstract semigroups (excluding vector spaces). The abbreviation $S G T$ means a semigroup with topology. So, a statement $\mathscr{S}$ is an $S G T$ means that $\mathscr{S}$ is both a semigroup and a (possibly non-Hausdorff) topological space, and no compatibility axiom (between these two structures) is assumed. In particular, in an SGT the binary action can be separately discontinuous. Although SGT's need not be Hausdorff, for us topological groups, by definition, are. An important example of non-topological SGT's are Ellis semigroups in which the binary action is continuous only in the left variable (according to the definition given in Chapter 6 of [1]). A classical example of compact Ellis semigroups that widely appear in functional analysis are the closed unit balls (considered as multiplicative semigroups with the weak* operator topologies) of the Banach spaces of the form $\mathscr{L}\left(X^{*}\right)$ where $X^{*}$ is the dual Banach space of a Banach space $X$ (only when $X$ is reflexive, the multiplication is separately continuous in these SGT's).

An SGT does not need to have a neutral element, and if it does have, it is said to be unital (otherwise it is called non-unital). Similarly, a homomorphism between two unital SGT's is called unital if it sends the unit of the source semigroup to the unit of the target.

By a right action of a semigroup $\mathscr{S}$ on a set $X$ we mean any function $X \times \mathscr{S} \ni$ $(x, s) \mapsto \phi_{s}(x)=x . s \in X$ such that for all $s, t \in \mathscr{S}$ and $x \in X,(x . s) . t=x$.(st). If, in addition,

- $\mathscr{S}$ is unital and $x .1=x$ for any $x \in X$, the action is called unital;

- $X$ is a convex set (in a real vector space) and $\phi_{s}$ is affine - that is,

$$
((1-\alpha) x+\alpha y) . s=(1-\alpha)(x . s)+\alpha(y . s)
$$

for any $x, y \in X$ and $\alpha \in[0,1]$, the action is called affine.

A point $a \in X$ is said to be a fixed point for the action if $a . s=a$ for any $s \in \mathscr{S}$.

When $(x, s) \mapsto x . s$ is a right action of $\mathscr{S}$ on a set $X$ and $u: X \rightarrow Y$ is an arbitrary function, for any $s \in \mathscr{S}, u_{s}: X \rightarrow Y$ is defined by $u_{s}(t)=u(t s)$. This, in particular, applies to the natural right action of $\mathscr{S}$ on itself.

For a topological space $X, C_{b}(X)$ stands for the algebra of all bounded continuous real-valued functions on $X$, equipped with the sup-norm. If $X$ is compact, we write $C(X)$ in place of $C_{b}(X)$. 
For any Banach space $E, \mathscr{L}(E)$ denotes the algebra of all bounded linear operators from $E$ into $E$. When $F$ is a closed linear subspace of $E$, a statement "P:E $\rightarrow F$ is a projection" means that $P$ is a bounded linear operator such that $P f=f$ for any $f \in F$. If $F$ is complemented in $E, \lambda(E, F)$ is used to denote the quantity specified in Theorem 1.1. A function $\mathscr{S} \ni s \mapsto T_{s} \in \mathscr{L}(E)$ is a homomorphism if $T_{s t}=T_{s} T_{t}$ for all $s, t \in \mathscr{S}$.

\section{Two KINDS OF AMENABILITY}

In the literature there are two classes of SGT's in which amenability is well studied - these are the classes of locally compact topological groups (consult, e.g., [7, 17, 18] or Chapter 1 in [22]) and of abstract semigroups (that is, semigroups without topologies or, equivalently, discrete semigroups; see, e.g., 17] or Section 1 in [7]). Amenability was also generalised to Banach algebras (see, e.g., Chapter 2 in [22] or 9] and references therein). Amenability in $C^{*}$-algebras also has a special interest (consult, e.g., [14]).

A topic closely related to amenable groups are extremely amenable Polish groups, which are now intensively studied. According to our Definition 3.1 (see below), all such groups are weakly right amenable. The literature on extremely amenable Polish groups is still growing up and we mention only a few of them: [8, [12, [19], [6], 23] and references therein or [16].

Because of further applications, we will deal with two versions of right amenability. Of course, in a similar manner one can introduce their counterparts for left amenability.

From now on, let $\mathscr{S}$ be an SGT and $C_{r}(\mathscr{S})$ consist of all functions $f \in C_{b}(\mathscr{S})$ such that $f_{s}$ is continuous for any $s \in \mathscr{S}$.

Let $\mathcal{F}$ be a linear subspace of $C_{r}(\mathscr{S})$. A mean on $\mathcal{F}$ is a linear functional $\phi: \mathcal{F} \rightarrow$ $\mathbb{R}$ such that $\inf f(\mathscr{S}) \leqslant \phi(f) \leqslant \sup f(\mathscr{S})$ for any $f \in \mathcal{F}$. (If $\mathcal{F}$ contains a function $j$ constantly equal to 1 , a linear functional $\phi$ on $\mathcal{F}$ is a mean iff $\|\phi\|=\phi(j)=1$.) The space $\mathcal{F}$ is said to be right invariant if $u_{s} \in \mathcal{F}$ for any $u \in \mathcal{F}$ and $s \in \mathscr{S}$. (It is easily seen that $C_{r}(\mathscr{S})$ is right invariant.)

A mean $\phi$ on a right invariant linear subspace $\mathcal{F}$ of $C_{r}(\mathscr{S})$ is right invariant if $\phi\left(f_{s}\right)=\phi(f)$ for any $f \in \mathcal{F}$ and $s \in \mathscr{S}$.

Right amenability deals with right invariant means and asks about their existence on suitably chosen right invariant linear spaces $\mathcal{F}$. The bigger this space $\mathcal{F}$ is, the better version of amenability we get. Below we propose two of them.

3.1. Definition. An SGT $\mathscr{S}$ is said to be weakly right amenable if there is a right invariant mean on

$$
\begin{aligned}
C_{\text {norm }}(\mathscr{S}) \stackrel{\text { def }}{=}\left\{f \in C_{r}(\mathscr{S}): s\right. & \mapsto f_{s} \text { and } s \mapsto f_{\text {st }} \text { are continuous } \\
& \text { in the norm topology of } \left.C_{r}(\mathscr{S}) \text { for all } t \in \mathscr{S}\right\} .
\end{aligned}
$$

is said to be strongly right amenable if there is a right invariant mean on

$$
C_{\text {weak }}(\mathscr{S}) \stackrel{\text { def }}{=}\left\{f \in C_{r}(\mathscr{S}): s \mapsto f_{s} \quad \text { and } \quad s \mapsto f_{s t} \quad\right. \text { are continuous }
$$

in the weak topology of $C_{r}(\mathscr{S})$ for all $\left.t \in \mathscr{S}\right\}$.

Since $C_{\text {norm }}(\mathscr{S}) \subset C_{\text {weak }}(\mathscr{S})$, a strongly right amenable SGT is weakly right amenable.

The following result, proved by Day [4, 5] (see Theorem 3 therein) for amenable abstract semigroups, is a powerful tool that makes amenable SGT's useful.

3.2. Theorem. For an SGT $\mathscr{S}$ the following conditions are equivalent: (SG1) $\mathscr{S}$ is weakly [resp. strongly] right amenable; 
(SG2) every jointly [resp. separately] continuous affine right action of $\mathscr{S}$ on a nonempty compact convex set in a Hausdorff locally convex topological vector space has a fixed point.

If, in addition, $\mathscr{S}$ is unital, then the above conditions are equivalent to:

(SG2') every jointly [resp. separately] continuous unital affine right action of $\mathscr{S}$ on a non-empty compact convex set in a Hausdorff locally convex topological vector space has a fixed point.

Here we postpone the proof of the above theorem to Section 5 where we give its sketch for the sake of completeness.

3.3. Examples. (A) Let $\mathscr{G}$ be a compact topological group. Then $C_{\text {weak }}(\mathscr{G})=$ $C_{\text {norm }}(\mathscr{G})=C(\mathscr{G})$ and $\mathscr{G}$ is strongly right amenable. The functional induced by the Haar measure of $\mathscr{G}$ is a unique invariant mean on $C(\mathscr{G})$.

(B) Every abelian semigroup $\mathscr{S}$ equipped with the discrete topology is strongly right amenable and thus $\mathscr{S}$ is such when equipped with totally arbitrary topology. This is a consequence of a well-known Markov-Kakutani fixed point theorem [15, [11. The fact that abelian SGT's are strongly right amenable will be used in the proof of all results formulated in Section 1.

(C) When the topology of an SGT $\mathscr{S}$ is discrete, $C_{\text {weak }}(\mathscr{S})=C_{\text {norm }}(\mathscr{S})=$ the algebra of all bounded real-valued functions on $\mathscr{S}$ and thus $\mathscr{S}$ is strongly right amenable iff it is weakly right amenable, iff it is right amenable as an abstract semigroup (considered without topology) 22.

(D) Let $\mathscr{G}$ be a locally compact topological group and $U C(\mathscr{G})$ stand for the algebra of all those functions $f \in C_{b}(\mathscr{G})$ that are uniformly continuous (cf. [22]). It can be easily shown that $U C(\mathscr{G}) \subset C_{\text {norm }}(\mathscr{G}) \subset C_{\text {weak }}(\mathscr{G}) \subset C_{b}(\mathscr{G})$ and thus, thanks to Theorem 1.1.9 in [22], $\mathscr{G}$ is strongly right amenable iff it is weakly right amenable, iff $\mathscr{G}$ is amenable in a classical sense (that is, if there is an invariant mean on $\left.L^{\infty}(\mathscr{G})\right)$.

(E) It is well known that the free (non-abelian) group $\mathscr{F}_{2}$ in two generators is nonamenable as an abstract group - or, equivalently, is not weakly right amenable when equipped with the discrete topology. It is also well known that there are two orthogonal matrices $U, V \in O_{3}$ that generate a group $\mathscr{G}$ isomorphic to $\mathscr{F}_{2}$. Since the closure $\overline{\mathscr{G}}$ of $\mathscr{G}$ (in $O_{3}$ ) is compact, $\overline{\mathscr{G}}$ is (strongly and thus) weakly right amenable. In Section 5 we will show that a dense subgroup of a weakly right amenable topological group is weakly right amenable as well (see Theorem 5.2). We conclude that $\mathscr{F}_{2}$ admits a separable metrizable topology that makes $\mathscr{F}_{2}$ a weakly right amenable topological group.

More on right amenability the reader can find in Section 5.

\section{Applications}

In this section we show how right amenability can be applied in operator theory. Although our first result has nothing in common with amenability, the method of its proof was one of our motivations.

4.1. Proposition. Let a closed linear subspace $Y$ of a Banach space $X$ and $T \in$ $\mathscr{L}(X)$ be such that there is a unique minimal projection $P$ of $X$ onto $Y, T(Y)=Y$, $T \uparrow Y$ is an isometry and $\|T\|=1$. Then $T P=P T$.

Proof. Observe that $Q \stackrel{\text { def }}{=}(T \uparrow Y)^{-1} P T$ is a projection onto $Y$ such that $\|Q\| \leqslant$ $\|P\|$. Since $P$ is unique minimal, we have $Q=P$ and hence $T P=P T$.

The idea of the above proof will be applied in the proof of the following 
4.2. Proposition. Let $\Phi: \mathscr{S} \ni s \mapsto T_{s} \in \mathscr{L}(X)$ be a homomorphism of a strongly [resp. weakly] right amenable $S G T \mathscr{S}$ into $\mathscr{L}(X)$ where $X$ is a Banach space. Suppose $Y$ is a closed linear subspace of $X$ and:

(p0) $\Phi$ is continuous in the strong operator topology [resp. operator norm topology] of $\mathscr{L}(X)$

(p1) for any $s \in \mathscr{S}, T_{s}(Y)=Y$ and the restriction $T_{s} \uparrow Y$ of $T_{s}$ to $Y$ is an isomorphism;

(p2) $Y$ is a dual Banach space; that is, $Y$ is linearly isometric to the dual space of some Banach space (and thus the closed unit ball $B_{Y}$ of $Y$ is compact with respect to certain locally convex topology, called further weak ${ }^{*}$, of $Y$ );

(p3) for any $s \in \mathscr{S}, T_{s} \uparrow Y$ is weak ${ }^{*}$ continuous on $B_{Y}$;

(p4) the function $\mathscr{S} \ni s \mapsto\left\|\left(T_{s} \uparrow Y\right)^{-1}\right\| \in \mathbb{R}$ is locally bounded.

Then the following conditions are equivalent:

(i) there exists a projection $P: X \rightarrow Y$ such that $P T_{s}=T_{s} P$ for all $s \in \mathscr{S}$;

(ii) there is a projection $Q: X \rightarrow Y$ such that

$$
\sup _{s \in \mathscr{S}}\left\|\left(T_{s} \uparrow Y\right)^{-1} Q T_{s}\right\|<\infty .
$$

Moreover, if (ii) holds, then there exists a projection $P_{0}: X \rightarrow Y$ such that $P_{0}$ commutes with all operators $T_{s}$ and $\left\|P_{0}\right\| \leqslant\|P\|$ for any other projection $P: X \rightarrow Y$ commuting with all $T_{s}$.

The part concerning a 'minimal' projection $P_{0}$ generalises a classical theorem due to Cheney and Morris [3] on the existence of minimal projections for complemented subspaces that are dual Banach spaces. It is worth noting here that the above condition (p4) is automatically fulfilled (thanks to (p0)) in the version of this proposition for weakly right amenable SGT's.

Proof of Proposition 4.2. First of all, (p3) combined with Krel̆n-Smulian theorem (that characterises weak* closed convex sets) implies that

(p3') for any $s \in \mathscr{S}, T_{s} \uparrow Y$ is a dual operator and $\left(T_{s} \uparrow Y\right)^{-1}$ is weak* continuous (on the whole $Y$ ).

For a projection $P: X \rightarrow Y$ and $s \in \mathscr{S}$ we write $P . s$ to denote $\left(T_{s} \uparrow Y\right)^{-1} P T_{s}$. Observe that P.s: $X \rightarrow Y$ is again a projection and that

$$
(P, s) \mapsto P . s
$$

is an affine right action of $\mathscr{S}$ on the set of all projection from $X$ onto $Y$.

Assume $Q$ is as specified in (ii). Put $M \stackrel{\text { def }}{=} \sup _{s \in \mathscr{S}}\|Q . s\|<\infty$. Let the set $\Delta$ of all projections $P: X \rightarrow Y$ with $\|P\| \leqslant M$ and $\sup _{s \in \mathscr{S}}\|P . s\| \leqslant M$ be equipped with the topology of pointwise weak* convergence (we call it weak* operator topology). It is easily seen that $\Delta$ is convex, Q.s $\in \Delta$ and $P . s \in \Delta$ for any $P \in \Delta$ and $s \in \mathscr{S}$. Moreover, it follows from (p3') that $\Delta$ is compact. So, by Theorem 3.2, it suffices to show that the action (4-2) is separately [resp. jointly] continuous.

It follows from (p3') that $\Delta \ni P \mapsto P . s \in \Delta$ is continuous for each $s \in \mathscr{S}$. Further, if $x$ is an arbitrary vector of $X$ and $\left(s_{\sigma}\right)_{\sigma \in \Sigma}$ is a net in $\mathscr{S}$ convergent to $s \in \mathscr{S}$, then $\lim _{\sigma \in \Sigma} T_{s_{\sigma}} x=T_{s} x$ and hence (thanks to $\left.(\mathrm{p} 4)\right)$ the nets $\left(T_{s_{\sigma}} x\right)_{\sigma \in \Sigma}$ and $\left(\left\|\left(T_{s_{\sigma}} \uparrow Y\right)^{-1}\right\|\right)_{\sigma \in \Sigma}$ are eventually bounded. This implies that $\left(T_{s_{\sigma}}\lceil Y)^{-1}\right.$ converge in the strong operator topology to $\left(T_{s} \uparrow Y\right)^{-1}$ and $\lim _{\sigma \in \Sigma}\left(P . s_{\sigma}\right) x=(P . s) x$ for every $P \in \Delta$. So, the action is separately continuous. Finally, assume that, in addition, $\mathscr{S}$ is weakly right amenable, and then-by $(\mathrm{p} 0)$ - we get

$$
\lim _{\sigma \in \Sigma}\left\|T_{s_{\sigma}}-T_{s}\right\|=0
$$


as well as $\lim _{\sigma \in \Sigma}\left\|\left(T_{s_{\sigma}} \uparrow Y\right)^{-1}-\left(T_{s} \uparrow Y\right)^{-1}\right\|=0$. Now if $\left(P_{\sigma}\right)_{\sigma \in \Sigma}$ is a net in $\Delta$ convergent (in the topology of $\Delta$ ) to $P \in \Delta$, then the net $\left(\left(T_{s_{\sigma}} \uparrow Y\right)^{-1} P_{\sigma}\right)_{\sigma \in \Sigma}$ is bounded and converges in the weak* operator topology to $\left(T_{s} \uparrow Y\right)^{-1} P$ (thanks to (p3') and the boundedness of $\Delta$ ), which - combined with (4-3) -implies that $P_{\sigma} . s_{\sigma}$ converge to P.s in the topology of $\Delta$.

To complete the proof, observe that the additional claim of the proposition follows from the compactness of $\Delta$ in the weak* operator topology, and from the lower semicontinuity of the operator norm in this topology.

4.3. Remark. Proposition 4.2 in the version for weak right amenability can easily be generalised (in a sense) to closed linear subspaces $Y$ of $X$ that are not dual Banach spaces. More precisely, if all assumptions of that result (for weak right amenability) hold, except for (p2) and (p3), and condition (ii) (therein) is fulfilled, then there exists a projection $P: X^{* *} \rightarrow Y^{\perp \perp}$ that commutes with all $T_{s}^{* *}$. Indeed, it suffices to observe that under the settings described above, all assumptions of Proposition 4.2 are satisfied for the homomorphism $s \mapsto T_{s}^{* *}$ (with $X$ and $Y$ replaced by $X^{* *}$ and $Y^{\perp \perp}$, respectively).

4.4. Definition. When $Y$ is a closed linear subspace of a Banach space $X$ and $\Phi: \mathscr{S} \ni s \mapsto T_{s} \in \mathscr{L}(X)$ is a homomorphism, a projection $P_{0}: X \rightarrow Y$ is called $\Phi$-minimal if $P_{0}$ commutes with all $T_{s}$, and $\left\|P_{0}\right\| \leqslant\|P\|$ for any other projection $P: X \rightarrow Y$ that commutes with all $T_{s}$.

When $Y$ is a finite-dimensional subspace, Proposition 4.2 can be strengthened as follows.

4.5. Proposition. Let $\Phi: \mathscr{S} \ni s \mapsto T_{s} \in \mathscr{L}(X)$ be a homomorphism of a strongly [resp. weakly] right amenable $S G T \mathscr{S}$ into $\mathscr{L}(X)$ where $X$ is a Banach space. Suppose $Y$ is a finite-dimensional linear subspace of $X$ and:

(fp0) $\Phi$ is continuous in the weak operator topology [resp. strong operator topology] of $\mathscr{L}(X)$;

(fp1) for any $s \in \mathscr{S}, T_{s}(Y)=Y$.

Then the following conditions are equivalent:

(i) there exists a projection $P: X \rightarrow Y$ such that $P T_{s}=T_{s} P$ for all $s \in \mathscr{S}$;

(ii) there is a projection $Q: X \rightarrow Y$ such that

$$
\sup _{s \in \mathscr{S}}\left\|\left(T_{s} \uparrow Y\right)^{-1} Q T_{s}\right\|<\infty .
$$

Moreover, if (ii) holds, then there exists a $\Phi$-minimal projection $P_{0}: X \rightarrow Y$.

The proof is quite similar to the previous one and thus we skip it. (Use the fact that under the assumptions of Proposition 4.5, both the functions

$$
\mathscr{S} \ni s \mapsto T_{s} \uparrow Y \in \mathscr{L}(Y) \quad \text { and } \quad \mathscr{S} \ni s \mapsto\left(T_{s} \uparrow Y\right)^{-1} \in \mathscr{L}(Y)
$$

are continuous in the operator norm topology of $\mathscr{L}(Y)$.)

Consequences of Propositions 4.2 and 4.5 follow. The following result (with a different proof) for compact topological groups can be found in 20] (see also Theorem 5.18 in [21]) with a slightly different settings.

4.6. Theorem. Let $\Phi: \mathscr{S} \ni s \mapsto T_{s} \in \mathscr{L}(X)$ be a homomorphism of a strongly [resp. weakly] right amenable $S G T \mathscr{S}$ into $\mathscr{L}(X)$ where $X$ is a Banach space. Suppose $Y$ is a closed linear subspace of $X$ such that conditions (p0)-(p3) of Proposition 4.2 are fulfilled and:

$\left(\mathrm{p} 4^{\prime}\right)$ the quantity $m_{Y}(\Phi) \stackrel{\text { def }}{=} \sup _{s \in \mathscr{S}}\left(\left\|T_{s}\right\| \cdot\left\|\left(T_{s} \uparrow Y\right)^{-1}\right\|\right)$ is finite. 
Then there exists a $\Phi$-minimal projection $P_{0}: X \rightarrow Y$ iff $Y$ is a complemented subspace of $X$. Moreover, if $Y$ is complemented, each $\Phi$-minimal projection $P: X \rightarrow Y$ satisfies

$$
\|P\| \leqslant m_{Y}(\Phi) \lambda(X, Y) .
$$

Proof. First of all, observe that (p4') implies (p4) (from Proposition 4.2): otherwise we would have a net $\left(s_{\sigma}\right)_{\sigma \in \Sigma} \subset \mathscr{S}$ convergent to some $s \in \mathscr{S}$ such that

$$
\lim _{\sigma \in \Sigma} \|\left(T_{s_{\sigma}}\lceil Y)^{-1} \|=\infty .\right.
$$

Then (p4') would imply that $\lim _{\sigma \in \Sigma}\left\|T_{s_{\sigma}}\right\|=0$ and consequently $T_{s}=0$, and $Y=\{0\}$ (by (p1)), which contradics (4-5).

Now thanks to Proposition 4.2, we only need to prove that a $\Phi$-minimal projection $P: X \rightarrow Y$ satisfies (4-4) provided $Y$ is complemented. By Cheney-Morris' theorem [3, there exists a projection $Q: X \rightarrow Y$ such that $\|Q\|=\lambda(X, Y)$. Now repeat the proof of Proposition 4.2 with $M \stackrel{\text { def }}{=} m_{Y}(\Phi) \lambda(X, Y)$ to conclude the existence of a projection $P: X \rightarrow Y$ that commutes with all $T_{s}$ and satisfies (4-4). The proof is complete.

The proof of the next result goes similarly as above and thus is omitted.

4.7. Theorem. Let $\Phi: \mathscr{S} \ni s \mapsto T_{s} \in \mathscr{L}(X)$ be a homomorphism of a strongly [resp. weakly] right amenable $S G T \mathscr{S}$ into $\mathscr{L}(X)$ where $X$ is a Banach space. Suppose $Y$ is a finite-dimensional linear subspace of $X$ such that conditions (fp0)(fp1) (of Proposition 4.2) and (p4') (of Theorem 4.6) hold. Then there exists a $\Phi$-minimal projection $P: X \rightarrow Y$, and $\|P\| \leqslant m_{Y}(\Phi) \lambda(X, Y)$.

For simplicity, for any homomorphism $\Phi: \mathscr{S} \rightarrow \mathscr{L}(X)$ (where $X$ is a Banach space) we denote by $\mathcal{N}(\Phi)$ and $\mathcal{R}(\Phi)$ the intersection of the kernels and, respectively, the closed linear span of the ranges of all operators of the form $\Phi(s)-I$ where $s \in \mathscr{S}$ and $I$ is the identity operator on $X$. When $\Phi$ is the identity function (and $\mathscr{S} \subset \mathscr{L}(X)$ ), we write $\mathcal{N}(\mathscr{S})$ and $\mathcal{R}(\mathscr{S})$ instead of $\mathcal{N}(\Phi)$ and $\mathcal{R}(\Phi)$.

4.8. Corollary. For an $S G T \mathscr{S}$ the following conditions are equivalent:

(a) $\mathscr{S}$ is strongly [resp. weakly] right amenable;

(b) for any homomorphism $\Phi: \mathscr{S} \rightarrow \mathscr{L}(X)$ continuous in the weak [resp. strong] operator topology with finite quantity $M(\Phi) \stackrel{\text { def }}{=} \sup _{s \in \mathscr{S}}\left\|T_{s}\right\|$ one has:

- $\mathcal{N}(\Phi) \cap \mathcal{R}(\Phi)=\{0\}$

- the subspace

$$
\mathcal{D}(\Phi) \stackrel{\text { def }}{=} \mathcal{N}(\Phi)+\mathcal{R}(\Phi)
$$

is closed in $X$, and

- the projection $P: \mathcal{D}(\Phi) \rightarrow \mathcal{N}(\Phi)$ induced by the decomposition (4-6) has norm not greater than $M(\Phi)$.

Proof. Assume (b) holds, put $X=C_{\text {norm }}(\mathscr{S})$ (resp. $X=C_{\text {weak }}(\mathscr{S})$ ) and define $\Phi: \mathscr{S} \ni s \mapsto T_{s} \in \mathscr{L}(X)$ by $T_{s} f=f_{s}$. Note that $\Phi$ is a homomorphism continuous in the weak (resp. strong) operator topology such that $M(\Phi)=1$. So, it follows from (b) that the projection $P: \mathcal{D}(\Phi) \rightarrow \mathcal{N}(\Phi)$ specified therein has norm not exceeding 1 . Observe that the function $j: \mathscr{S} \rightarrow \mathbb{R}$ constantly equal to 1 belongs to $\mathcal{N}(\Phi)$ and thus $1=\|j\|=\|P(j+z)\| \leqslant\|j+z\|$ for any $z \in \mathcal{R}(\Phi)$. Equivalently, $\operatorname{dist}(j, \mathcal{R}(\Phi))=1$. Now the Hahn-Banach theorem implies that there is a linear functional $\phi: X \rightarrow \mathbb{R}$ that vanishes on $\mathcal{R}(\Phi)$ and satisfies $\phi(j)=1=\|\phi\|$. This means that $\phi$ is an invariant mean of $X$ and we are done.

Now assume (a) holds and let $X$ and $\Phi: \mathscr{S} \ni s \mapsto T_{s} \in \mathscr{L}(X)$ be as specified in (b). Fix a non-zero vector $y \in \mathcal{N}(\Phi)$ and consider the linear subspace $Y$ generated 
by $y$. Since $Y$ is one-dimensional, $\lambda(X, Y)=1$. Observe that conditions (fp0) and (fp1) hold, and $m_{Y}(\Phi)=M(\Phi)$. So, Theorem 4.7 gives us a $\Phi$-minimal projection $P_{0}: X \rightarrow Y$ with $\left\|P_{0}\right\| \leqslant M(\Phi)$. For any $s \in \mathscr{S}$ and $x \in X$ we have

$$
P_{0}\left(T_{s} x-x\right)=T_{s}\left(P_{0} x\right)-P_{0} x=0,
$$

because $P_{0} x \in Y \subset \mathcal{N}(\Phi)$. So, $T_{s} x-x \in \operatorname{ker}\left(P_{0}\right)$ and consequently $\mathcal{R}(\Phi) \subset \operatorname{ker}\left(P_{0}\right)$. We conclude that for any $z \in \mathcal{R}(\Phi),\|y\|=\left\|P_{0}(y+z)\right\| \leqslant M(\Phi)\|y+z\|$. It follows from the arbitrariness of $y$ and $z$ that $\mathcal{N}(\Phi) \cap \mathcal{R}(\Phi)=\{0\}$ and $\|P\| \leqslant M(\Phi)$ (where $P$ is as specified in (b)). The closedness of $\mathcal{N}(\Phi)+\mathcal{R}(\Phi)$ follows from the continuity of $P$ and the closedness of both $\mathcal{N}(\Phi)$ and $\mathcal{R}(\Phi)$.

Recall that a Banach space $X$ is HI (hereditary indecomposable) if for every projection $P: Y \rightarrow Z$ where $Z \subset Y \subset X$ the kernel or the range of $P$ is finitedimensional.

As an immediate consequence of Corollary 4.8 (see item (B) in Examples 3.3) we obtain

4.9. Corollary. Let $\mathscr{T} \subset \mathscr{L}(E)$ be a bounded abelian multiplicative semigroup of operators on a HI Banach space $E$. Then either $\mathcal{N}(\mathscr{T})$ or $\mathcal{R}(\mathscr{T})$ is finitedimensional.

Now we offer a result on extending intertwining operators that reads as follows.

4.10. Theorem. Let $\Phi: \mathscr{S} \ni s \mapsto A_{s} \in \mathscr{L}(X)$ and $\Psi: \mathscr{S} \ni s \mapsto B_{s} \in \mathscr{L}(Y)$ be two homomorphisms of a strongly [resp. weakly] right amenable $S G T \mathscr{S}$ into $\mathscr{L}(X)$ and $\mathscr{L}(Y)$ where $X$ and $Y$ are Banach spaces. Suppose $T_{0}: E \rightarrow Y$ is a bounded linear operator from a closed linear subspace $E$ of $X$ into $Y$ and:

(i0) $\Phi$ and $\Psi$ are continuous in the strong operator topologies [resp. operator norm topologies] of $\mathscr{L}(X)$ and $\mathscr{L}(Y)$, respectively;

(i1) for any $s \in \mathscr{S}, A_{s}(E) \subset E$ and $B_{s}$ is an isomorphism;

(i2) $Y$ is a dual Banach space;

(i3) for any $s \in \mathscr{S}, B_{s}$ is weak* continuous on the closed unit ball $B_{Y}$ of $Y$;

(i4) the function $\mathscr{S} \ni s \mapsto\left\|B_{s}^{-1}\right\| \in \mathbb{R}$ is locally bounded;

(i5) $B_{s} T_{0}=T_{0} A_{s} \uparrow E$ for any $s \in \mathscr{S}$.

Then the following conditions are equivalent:

(i) $T_{0}$ extends to a bounded linear operator $T: X \rightarrow Y$ that intertwines $\Phi$ and $\Psi$, that is, $B_{s} T=T A_{s}$ for all $s \in \mathscr{S}$;

(ii) there exists a bounded linear operator $T^{\prime}: X \rightarrow Y$ that extends $T_{0}$ and satisfies $\sup _{s \in \mathscr{S}}\left\|B_{s}^{-1} T^{\prime} A_{s}\right\|<\infty$.

Proof. The proof is similar to that of Proposition 4.2 and thus we only give its sketch. For any bounded linear operator $L: X \rightarrow Y$ and $s \in \mathscr{S}$ we denote by $L . s$ the operator $B_{s}^{-1} L A_{s}$. This defines an affine right action of $\mathscr{S}$. Next, assume $T^{\prime}$ is as specified in (ii) and set $M \stackrel{\text { def }}{=} \sup _{s \in \mathscr{S}}\left\|T^{\prime} . s\right\|$. Equip the set $\Delta$ of all linear extensions $T: X \rightarrow Y$ of $T_{0}$ such that $\|T\| \leqslant M$ and $\sup _{s \in \mathscr{S}}\|T . s\| \leqslant M$ with the weak* operator topology. Now it suffices to repeat the arguments presented in the proof of Proposition 4.2 to conclude that the action defined above is suitably continuous on $\Delta \times \mathscr{S}$ and thus has a fixed point $T$ in $\Delta$. Then automatically $B_{s} T=T A_{s}$ for any $s \in \mathscr{S}$ and we are done.

The following result is a special case of Theorem 4.10 and we skip its proof.

4.11. Corollary. Suppose all assumptions of Theorem 4.10 (on $\Phi, \Psi, Y$ and $T_{0}$ ) hold. If, in addition, $\sup _{s \in \mathscr{S}}\left(\left\|B_{s}^{-1}\right\| \cdot\left\|A_{s}\right\|\right)<\infty$, then $T_{0}$ extends to a bounded linear operator $T: X \rightarrow Y$ intertwining $\Phi$ and $\Psi$ iff $T_{0}$ extends to a bounded linear operator from $X$ into $Y$. 
4.12. Example. As the following simple example shows, the assumption in Theorem 4.6 (and hence also in Proposition 4.2) that $T_{s}(Y)=Y$ for all $s \in \mathscr{S}$ (see (p1)) cannot be dropped in general. Let $X$ be a separable Hilbert space with an orthonormal basis $e_{0}, e_{1}, e_{2}, \ldots$ and $S: X \rightarrow X$ be the shift - that is, $S e_{n}=e_{n+1}(n \geqslant 0)$. Further, let $\mathscr{S}$ be the additive group of all natural numbers, $\Phi: \mathscr{S} \ni n \mapsto S^{n} \in \mathscr{L}(X)$ and $Y$ be the range of $S$. Then all assumptions of Theorem 4.6. except for " $S(Y)=Y$ ", are fulfilled and $Y$ is complemented. However, there is no projection onto $Y$ that commutes with $S$.

The above example can also be employed to show that the assumption in Corollary 4.11 that all the operators $B_{s}$ are isomorphisms (see (i1)) cannot be dropped in general. Indeed, it suffices to set, in addition to the above settings, $T_{0}$ as the identity operator on $E \stackrel{\text { def }}{=} Y \subset X$, and $\Psi=\Phi$.

Further results of this section deal with renorming-like issues. The first of them generalises results of Koehler and Rosenthal [13] (see therein for the definition of a semi-inner product on a Banach space).

4.13. Theorem. Let $\mathscr{S} \ni s \mapsto T_{s} \in \mathscr{L}(X)$ be a homomorphism of a weakly right amenable $S G T \mathscr{S}$ into $\mathscr{L}(X)$ (where $\left(X,\|\cdot\|_{X}\right)$ is a Banach space) that is continuous in the strong operator topology of $\mathscr{L}(X)$. Further, let $m$ and $M$ be two positive real constants such that

$$
m\|x\|_{X} \leqslant\left\|T_{s} x\right\|_{X} \leqslant M\|x\|_{X} \quad(x \in X, s \in \mathscr{S}) .
$$

Then there exists a norm $\|\cdot\|_{*}$ on $X$ such that for any $x \in X$ and $s \in \mathscr{S}, m\|x\|_{X} \leqslant$ $\|x\|_{*} \leqslant M\|x\|_{X}$ and $\left\|T_{s} x\right\|_{*}=\|x\|_{*}$.

If, in addition, the topology of $\mathscr{S}$ is discrete, then there exists a semi-inner product $[\cdot,-]_{*}$ inducing the norm $\|\cdot\|_{*}$ such that $\left[T_{s} x, T_{s} y\right]_{*}=[x, y]_{*}$ for all $x, y \in X$ and $s \in \mathscr{S}$.

Proof. For any seminorm $\|\cdot\|$ on $X$ and $s \in \mathscr{S}$ we denote by $\|\cdot\| . s$ the seminorm on $X$ that assigns to a vector $x$ the number $\left\|T_{s} x\right\|$. It is easy to check that

$$
(\|\cdot\|, s) \mapsto\|\cdot\| . s
$$

is an affine right action of $\mathscr{S}$ on the set of all seminorms on $X$.

Firstly, let $\Delta$ be the set of all norms $\|\cdot\|$ on $X$ such that $m\|x\|_{X} \leqslant\|x\| \leqslant M\|x\|_{X}$ and $m\|x\|_{X} \leqslant\left\|T_{s} x\right\| \leqslant M\|x\|_{X}$ for all $x \in X$ and $s \in \mathscr{S}$. We equip $\Delta$ with the pointwise convergence topology. Observe that $\Delta$ is convex and compact (by the Tychonoff theorem), and $\|\cdot\|_{X} . s \in \Delta$ (by (4-7) $)$ and $\|\cdot\| . s \in \Delta$ for all $\|\cdot\| \in \Delta$ and $s \in \mathscr{S}$. One also easily verifies that the action (4-8) is jointly continuous on $\Delta \times \mathscr{S}$. Hence, by Theorem 3.2, there is a norm $\|\cdot\|_{*} \in \Delta$ which is a fixed point for this action. This means that all $T_{s}$ are isometries with respect to this norm.

Secondly, let $\Gamma$ consist of all semi-inner products on $X$ that induce the norm $\|\cdot\|_{*}$. As before, $\Gamma$ is convex, compact (in the pointwise convergence topology) and non-empty. For $[\cdot,-] \in \Gamma$ and $s \in \mathscr{S}$, we define $[\cdot,-] . s \in \Gamma$ by $[x, y] . s \stackrel{\text { def }}{=}\left[T_{s} x, T_{s} y\right]$. As before, one easily checks that in this way we have defined an affine right action of $\mathscr{S}$ on $\Gamma$. So, if the topology of $\mathscr{S}$ is discrete, the fixed point property formulated in Theorem 3.2 finishes the proof.

4.14. Corollary. Let $\mathscr{S} \ni s \mapsto T_{s} \in \mathscr{L}(X)$ be a homomorphism of an $S G T \mathscr{S}$ into $\mathscr{L}(X)$ (where $(X,\|\cdot\|)$ is a Banach space) that is continuous in the strong operator topology of $\mathscr{L}(X)$. Further, assume $\mathscr{S}_{0}$ is a weakly right amenable subsemigroup of $\mathscr{S}$ (in the topology inherited from $\mathscr{S}$ ) such that

(*) $\mathscr{S}$ is a unique subsemigroup $\mathscr{Z}$ of $\mathscr{S}$ that is closed, contains $\mathscr{S}_{0}$ and has the following property: if $x, y \in \mathscr{S}$ and $x, x y \in \mathscr{Z}$, then $y \in \mathscr{Z}$. 
If $m$ and $M$ are two positive real constants such that

$$
m\|u\| \leqslant\left\|T_{s} u\right\| \leqslant M\|u\| \quad\left(u \in X, s \in \mathscr{S}_{0}\right),
$$

then for any $s \in \mathscr{S}$ and $u \in X$,

$$
\frac{m}{M}\|u\| \leqslant\left\|T_{s} u\right\| \leqslant \frac{M}{m}\|u\| .
$$

Proof. It follows from Theorem 4.13 that there exists a norm $\|\cdot\|_{*}$ on $X$ such that $m\|u\| \leqslant\|u\|_{*} \leqslant M\|u\|$ and $\left\|T_{s} u\right\|_{*}=\|u\|_{*}$ (that is, $T_{s}$ is isometric with respect to $\left.\|\cdot\|_{*}\right)$ for any $s \in \mathscr{S}_{0}$ and $u \in X$. Now let $\mathscr{Z}$ consist of all $s \in \mathscr{S}$ such that $T_{s}$ is isometric with respect to $\|\cdot\|_{*}$. We conclude from $(\star)$ that $\mathscr{Z}=\mathscr{S}$. But then, for any $s \in \mathscr{S}$ and $u \in X,\left\|T_{s} u\right\| \leqslant \frac{1}{m}\left\|T_{s} u\right\|_{*}=\frac{1}{m}\|u\|_{*} \leqslant \frac{M}{m}\|u\|$ and similarly $\left\|T_{s} u\right\| \geqslant \frac{1}{M}\left\|T_{s} u\right\|_{*}=\frac{1}{M}\|u\|_{*} \geqslant \frac{m}{M}\|u\|$.

4.15. Remark. The above result gives us some insight into the algebraic structure of a right amenable SGT and tells us how far it is from being free. If, for example, $\mathscr{S}_{0}$ is a weakly right amenable SGT that generates a topological group $\mathscr{G}$ and $\mathscr{G} \ni g \mapsto T_{g} \in \mathscr{L}(X)$ is a unital homomorphism that is continuous in the strong operator topology and satisfies (4-9) (see Corollary 4.14), then the operators of the form $T_{s_{1}}^{\varepsilon_{1}} \cdot \ldots \cdot T_{s_{k}}^{\varepsilon_{k}}$ (where $s_{1}, \ldots, s_{k} \in \mathscr{S}_{0}, \varepsilon_{1}, \ldots, \varepsilon_{k} \in\{-1,1\}$ and $k>0$ are arbitrary) are uniformly bounded.

We end this section with the next two generalisations of the Sz.-Nagy theorem 24]. Below we use $I$ to denote the identity operator on a Hilbert space $H$.

4.16. Proposition. Let $\mathscr{S} \ni s \mapsto T_{s} \in \mathscr{L}(H)$ be a homomorphism of a weakly right amenable $S G T \mathscr{S}$ into $\mathscr{L}(H)$ (where $(H,\langle\cdot,-\rangle)$ is a Hilbert space) that is continuous in the strong operator topology of $\mathscr{L}(H)$. Further, suppose there exist an operator $A_{0} \in \mathscr{L}(H)$ and two functions $m, M: H \rightarrow(0, \infty)$ such that for any $x \in H$,

$$
m(x)\|x\| \leqslant\left\|A_{0} T_{s} x\right\| \leqslant M(x)\|x\| \quad(s \in \mathscr{S}) .
$$

Then there exist a positive operator $A \in \mathscr{L}(H)$ with trivial kernel and a homomorphism $\mathscr{S} \ni s \mapsto V_{s} \in \mathscr{L}(H)$ continuous in the strong operator topology such that

- $m(x)\|x\| \leqslant\|A x\| \leqslant M(x)\|x\|$ for any $x \in H$;

- $A T_{s}=V_{s} A$ for all $s \in \mathscr{S}$;

- $V_{s}$ is an isometry for any $s \in \mathscr{S}$.

Proof. It follows from (4-10) and the Uniform Boundedness Principle that

$$
\sup _{s \in \mathscr{S}}\left\|A_{0} T_{s}\right\|<\infty \text {. }
$$

Consequently, we may and do assume that

$$
\sup _{x \in H} M(x)<\infty .
$$

For any $B \in \mathscr{L}(H)$ and $s \in \mathscr{S}$ let $B . s$ stand for the operator $T_{s}^{*} B T_{s}$. It is easily seen that $(B, s) \mapsto B . s$ is an affine right action of $\mathscr{S}$ on $\mathscr{L}(H)$.

Denote by $\Delta$ the set of all positive operators $B \in \mathscr{L}(H)$ such that

$$
m(x)^{2}\|x\|^{2} \leqslant\langle B x, x\rangle \leqslant M(x)^{2}\|x\|^{2}
$$

as well as

$$
m(x)^{2}\|x\|^{2} \leqslant\langle(B . s) x, x\rangle \leqslant M(x)^{2}\|x\|^{2}
$$

for any $x \in H$ and $s \in \mathscr{S}$, and equip $\Delta$ with the weak operator topology. It is clear that $\Delta$ is convex and compact, and that $\left(A_{0}^{*} A_{0}\right) . s \in \Delta$ (by (4-10) $)$ and B.s $\in \Delta$ for 
any $B \in \Delta$ and $s \in \mathscr{S}$. We claim that the action just defined is jointly continuous on $\Delta \times \mathscr{S}$. To be convinced of that, assume $\left(B_{\sigma}\right)_{\sigma \in \Sigma} \subset \Delta$ and $\left(s_{\sigma}\right)_{\sigma \in \Sigma} \subset \mathscr{S}$ are two nets that converge to, respectively, $B \in \Delta$ and $s \in \mathscr{S}$. Then $T_{s_{\sigma}}$ converge in the strong operator topology to $T_{s}$. Since the operators $B_{\sigma}$ are uniformly bounded (by (4-11), (4-12) and (4-13)), we conclude that $B_{\sigma} T_{s_{\sigma}}$ converge to $B T_{s}$ in the weak operator topology. So, when $x, y \in H$ are fixed, the net $\left(T_{s_{\sigma}} x\right)_{\sigma \in \Sigma}$ is eventually bounded and thus

$$
\lim _{\sigma \in \Sigma}\left\langle T_{s_{\sigma}}^{*} B_{\sigma} T_{s_{\sigma}} x, y\right\rangle=\lim _{\sigma \in \Sigma}\left\langle B_{\sigma} T_{s_{\sigma}} x, T_{s_{\sigma}} y\right\rangle=\left\langle B T_{s} x, T_{s} y\right\rangle=\left\langle T_{s}^{*} B T_{s} x, y\right\rangle
$$

(because the vectors $B_{\sigma} T_{s_{\sigma}} x$ are uniformly bounded and converge weakly to $B T_{s} x$, and $T_{s_{\sigma}} y$ converge in the norm to $\left.T_{s} y\right)$.

Now the fixed point property from Theorem 3.2 gives us an operator $B \in \Delta$ such that

$$
T_{s}^{*} B T_{s}=B \quad(s \in \mathscr{S}) .
$$

We define $A$ as the (positive) square root of $B$. For any $x \in H,\|A x\|^{2}=\langle B x, x\rangle$ and thus $m(x)\|x\| \leqslant\|A x\| \leqslant M(x)\|x\|$ (thanks to (4-12) $)$, which in turn implies that the kernel of $A$ is trivial.

Fix for a moment $s \in \mathscr{S}$ and let $V_{s}$ be the partial isometry that appears in the polar decomposition $A T_{s}=V_{s}\left|A T_{s}\right|$ (where $\left|A T_{s}\right|=\sqrt{\left(A T_{s}\right)^{*}\left(A T_{s}\right)}$ ) of the operator $A T_{s}$. The equation (4-14) gives $\left(A T_{s}\right)^{*}\left(A T_{s}\right)=A^{2}$. So, by the uniqueness of the positive square root we obtain $\left|A T_{s}\right|=A$ and consequently $A T_{s}=V_{s} A$. Since $A$ has trivial kernel, $V_{s}$ is an isometry.

It remains to check that $s \mapsto V_{s}$ is a homomorphism continuous in the strong operator topology. For $s, t \in \mathscr{S}$ we have $V_{s t} A=A T_{s t}=A T_{s} T_{t}=V_{s} A T_{t}=V_{s} V_{t} A$. Since the range of $A$ is dense in $H$, we get $V_{s t}=V_{s} V_{t}$. Finally, if $s_{\sigma} \in \mathscr{S}$ converge to $s \in \mathscr{S}$, then for any $x \in H, \lim _{\sigma \in \Sigma} V_{s_{\sigma}}(A x)=\lim _{\sigma \in \Sigma} A\left(T_{s_{\sigma}} x\right)=A T_{s} x=V_{s}(A x)$ and, again, the density of the range of $A$ in $H$ implies that $V_{s_{\sigma}}$ converge to $V_{s}$ in the strong operator topology.

As an immediate consequence of the above result, we obtain

4.17. Corollary. Let $\mathscr{S} \ni s \mapsto T_{s} \in \mathscr{L}(H)$ be a homomorphism of a weakly right amenable $S G T \mathscr{S}$ into $\mathscr{L}(H)$ (where $H$ is a Hilbert space) that is continuous in the strong operator topology of $\mathscr{L}(H)$. There exists an invertible positive operator $A \in \mathscr{L}(H)$ such that $A T_{s} A^{-1}$ is an isometry for each $s \in \mathscr{S}$ iff there are two positive real constants $m$ and $M$ such that

$$
m\|x\| \leqslant\left\|T_{s} x\right\| \leqslant M\|x\| \quad(s \in \mathscr{S}, x \in H) .
$$

Moreover, if (4-15) holds, the above operator $A$ can be chosen so that $m I \leqslant A \leqslant$ $M I$.

The proof is left to the reader.

4.18. Remark. Corollary 4.17 combined with Corollary 4.14 leads to a stronger generalisation of the Sz.-Nagy theorem that reads as follows:

If $\mathscr{S} \ni s \mapsto T_{s} \in \mathscr{L}(H)$ is a homomorphism of an $S G T \mathscr{S}$ into $\mathscr{L}(H)$ (where $H$ is a Hilbert space) that is continuous in the strong operator topology and there are a weakly right amenable subsemigroup $\mathscr{S}_{0}$ of $\mathscr{S}$ and two positive real constants $m$ and $M$ such that condition $(\star)$ of Corollary 4.14 is fulfilled and the inequality $m\|x\| \leqslant\left\|T_{s} x\right\| \leqslant M\|x\|$ holds for any $s \in \mathscr{S}_{\mathbf{0}}$ and $x \in H$, then there exists an invertible positive operator $A \in \mathscr{L}(H)$ such that $A T_{s} A^{-1}$ is an isometry for each $s \in \mathscr{S}$. 
In particular, if $\mathscr{G}$ is a topological group and a weakly right amenable semigroup $\mathscr{S} \subset \mathscr{G}$ generates $\mathscr{G}$, then each unital homomorphism $\Phi: \mathscr{G} \ni g \mapsto T_{g} \in \mathscr{L}(H)$ that is continuous on $\mathscr{S}$ in the strong operator topology and satisfies condition (4-15) of Corollary 4.17 has image $\Phi(\mathscr{G})$ "similar" to a subgroup of the unitary group of $H$.

Proofs of Theorems 1.1, 1.2, 1.3 and 1.4. As all discrete abelian semigroups are strongly right amenable (see item (B) in Examples 3.3), the theorems of the first section are special cases of the results of this part.

\section{More on amenability}

Proof of Theorem 3.2. Let $\mathcal{F} \stackrel{\text { def }}{=} C_{\text {norm }}(\mathscr{S})\left[\operatorname{resp} . \mathcal{F} \stackrel{\text { def }}{=} C_{\text {weak }}(\mathscr{S})\right]$.

First assume (SG2) (or (SG2')) holds. Let the set $\Delta$ of all means on $\mathcal{F}$ be equipped with the weak ${ }^{*}$ topology. For $\phi \in \Delta$ and $s \in \mathscr{S}$ we define $\phi . s \in \Delta$ by $(\phi . s)(f)=\phi\left(f_{s}\right)$. Then $(\phi, s) \mapsto \phi . s$ is a (unital if $\mathscr{S}$ is unital) affine right action that is jointly [resp. separately] continuous. So, an application of (SG2) (or (SG2')) yields the existence of a right invariant mean.

Now assume $\mathscr{S}$ is right amenable and let $K$ and $K \times \mathscr{S} \ni(x, s) \mapsto x . s \in K$ be, respectively, a non-empty compact convex set in a Hausdorff locally convex topological vector space and a jointly [resp. separately] continuous affine right action. Denote by $C A(K) \subset C(K)$ and $j$, respectively, the Banach space of all real-valued continuous affine functions on $K$ and the function from $C A(K)$ constantly equal to 1. Fix a right invariant mean $\phi$ on $\mathcal{F}$ and a point $b \in K$. For each $u \in C A(K)$ let $\hat{u} \in \mathcal{F}$ be given by $\hat{u}(s)=u(b . s)$. Observe that $\Lambda: C A(K) \ni u \mapsto \phi(\hat{u}) \in \mathbb{R}$ is a linear functional such that $\Lambda(j)=1=\|\Lambda\|$. It follows from a well-known characterisation of such functionals on $C A(K)$ that there is $a \in K$ for which $\Lambda(u)=u(a)$ for any $u \in C A(K)$. In order to check that $a$ is a fixed point for the action, it suffices to verify that $u(a . s)=u(a)$ for any $u \in C A(K)$. To this end, note that $\hat{u}_{s}=\widehat{u_{s}}$ for any such a function $u$ and all $s \in \mathscr{S}$. But then $u(a . s)=u_{s}(a)=\Lambda\left(u_{s}\right)=\phi\left(\widehat{u_{s}}\right)=\phi\left(\hat{u}_{s}\right)=\phi(\hat{u})=\Lambda(u)=u(a)$ and we are done.

To formulate our next result, let us introduce some elementary notion. For any (unital or non-unital) SGT $\mathscr{S}$ we use $\mathscr{S}^{\sqcup 1}$ to denote its unitization; that is, $\mathscr{S}^{\sqcup 1}$ is a unital SGT such that $\mathscr{S}^{\sqcup 1}=\mathscr{S} \sqcup\{1\}$ where $1 \notin \mathscr{S}$ is the neutral element of $\mathscr{S}^{\sqcup 1}$ as well as its isolated point, the multiplication of $\mathscr{S}^{\sqcup 1}$ extends that in $\mathscr{S}$, $\mathscr{S}$ is both closed and open in $\mathscr{S}^{\sqcup 1}$ and the topology of $\mathscr{S}$ coincides with the one inherited from $\mathscr{S}^{\sqcup 1}$. Note that even if $\mathscr{S}$ is unital, the neutral element of $\mathscr{S}^{\sqcup 1}$ differs from the one of $\mathscr{S}$.

Below we list basic properties of the class of right amenable SGT's.

5.1. Proposition. (A) An SGT $\mathscr{S}$ is weakly [resp. strongly] right amenable iff so is $\mathscr{S}^{\sqcup 1}$

(B) Let $h: \mathscr{T} \rightarrow \mathscr{S}$ be a continuous onto homomorphism between SGT's. If $\mathscr{T}$ is weakly [resp. strongly] right amenable, so is $\mathscr{S}$.

(C) Let $\mathscr{T}$ be a subsemigroup of an $S G T \mathscr{S}$ such that $\mathscr{S}$ is a unique subsemigroup $\mathscr{Z}$ of $\mathscr{S}$ that is closed, contains $\mathscr{T}$ and has the following property:

$(\star \star)$ if $x, y \in \mathscr{S}$ and $x, x y \in \mathscr{Z}$, then $y \in \mathscr{Z}$.

Then, if $\mathscr{T}$ is weakly [resp. strongly] right amenable, so is $\mathscr{S}$.

(D) Let $\left\{\mathscr{S}_{b}\right\}_{b \in B}$ be a family of subsemigroups of an $S G T \mathscr{S}$ such that $\mathscr{S}$ coincides with the smallest closed subsemigroup that contains $\bigcup_{b \in B} \mathscr{S}_{b}$. Assume ss ${ }^{\prime}=$ $s^{\prime} s$ for any $s \in \mathscr{S}_{b}$ and $s^{\prime} \in \mathscr{S}_{b^{\prime}}\left(b, b^{\prime} \in B\right)$ with $b \neq b^{\prime}$. If all $\mathscr{S}_{b}$ are weakly [resp. strongly] right amenable, so is $\mathscr{S}$. 
(E) The (topological) product of a family of weakly [resp. strongly] right amenable unital SGT's is weakly [resp. strongly] right amenable as well.

We do not know whether the assumption in (E) that SGT's are unital can be dropped (even when the family of SGT's is finite).

Proof of Proposition 5.1. Item (A) is left to the reader as a simple exercise. To prove each of (B), (C) and (D), fix a jointly [resp. separately] continuous affine right action

$$
K \times \mathscr{S} \ni(x, s) \mapsto x . s \in K
$$

of $\mathscr{S}$ on a non-empty compact convex set $K$. Under the settings of (B), also $K \times \mathscr{T} \ni(x, t) \mapsto x . h(t) \in K$ is such an action. So, Theorem 3.2 implies that this last action has a fixed point. It follows from the surjectivity of $h$ that also (5-1) has a fixed point, and we are done. Under the settings of (C), there is $a \in K$ such that $a . t=a$ for any $t \in \mathscr{T}$. Since $\mathscr{Z} \stackrel{\text { def }}{=}\{s \in \mathscr{S}: a . s=a\}$ is a closed subsemigroup of $\mathscr{S}$ that contains $\mathscr{T}$ and satisfies $(\star \star)$, the assumptions of $(\mathrm{C})$ imply that $\mathscr{S}=\mathscr{Z}$, which means that, again, (5-1) has a fixed point.

We turn to (D). For any $b \in B$ set $F_{b} \stackrel{\text { def }}{=}\left\{x \in K: x . s=x\right.$ for any $\left.s \in \mathscr{S}_{b}\right\}$. It follows that $F_{b}$ is compact, convex, and non-empty-by a suitable amenability of $\mathscr{S}_{b}$. We claim that $L \stackrel{\text { def }}{=} \bigcap_{b \in B} F_{b}$ is non-empty as well. To be convinced of that, it is enough to check that $\bigcap_{k=1}^{n} F_{b_{k}} \neq \varnothing$ for any $b_{1}, \ldots, b_{n} \in B$ (and $n>0$ ). We proceed by induction on $n$. The case $n=1$ has already been established. Now if $n>1$ and $b_{1}, \ldots, b_{n} \in B$ are arbitrary, then $D \stackrel{\text { def }}{=} \bigcap_{k=1}^{n-1} F_{b_{k}}$ is compact, convex, and nonempty (by the induction hypthesis). Moreover, if $s \in \mathscr{S}_{b_{n}}$ and $x \in D$, then $x . s \in D$. (Indeed, if $k<n$ and $t \in \mathscr{S}_{b_{k}}$, then $(x . s) . t=x .(s t)=x .(t s)=(x . t) . s=x . s$.) So, $D \times \mathscr{S}_{b_{n}} \ni(x, s) \mapsto x . s \in D$ is a correctly defined action that has a fixed point, by Theorem 3.2 Equivalently, $D \cap F_{b_{n}} \neq \varnothing$, which finishes the proof that $L$ is nonempty. Now let $a \in L$. The set $\mathscr{T} \stackrel{\text { def }}{=}\{s \in \mathscr{S}: a . s=a\}$ is a closed subsemigroup of $\mathscr{S}$ that contains $\bigcup_{b \in B} \mathscr{S}_{b}$ and hence $\mathscr{T}=\mathscr{S}$, thanks to the assumptions of (D).

Finally, we turn to (E). So, assume $\mathscr{S}$ is the product of suitably amenable unital SGT's $\mathscr{T}_{b}(b \in B)$ and for all $b \in B$ denote by $e_{b}$ the neutral element of $\mathscr{T}_{b}$. Further, for any $d \in B$ and $t \in \mathscr{T}_{d}$ denote by $t^{(d)}$ the element $\left(z_{b}\right)_{b \in B} \in \mathscr{S}$ such that $z_{b}=e_{b}$ for $b \neq d$ and $z_{d}=t$, and set $\mathscr{S}_{d} \stackrel{\text { def }}{=}\left\{x^{(d)}: x \in \mathscr{T}_{d}\right\}$. Since $\mathscr{T}_{d} \ni x \mapsto x^{(d)} \in \mathscr{S}_{d}$ is a continuous surjective homomorphism, it follows from item (B) that $\mathscr{S}_{d}$ is suitably amenable. Now it suffices to observe that for $\left\{\mathscr{S}_{b}\right\}_{b \in B}$ and $\mathscr{S}$ all assumptions of item (D) are satisfied and apply this part.

The next property (in the class of topological groups), with which we end the paper, is less elementary.

5.2. Theorem. If an SGT $\mathscr{S}$ contains a dense subsemigroup that is weakly [resp. strongly] right amenable, then $\mathscr{S}$ itself is weakly [resp. strongly] right amenable.

A dense subgroup of a weakly right amenable topological group is weakly right amenable as well.

Proof. The first claim of the theorem is a special case of item (C) of Proposition 5.1.

To prove the second claim, we will make use of so-called Raukov-complete topological groups (see, e.g., Section 3.6 in [2]). We need the following two facts about them:

(RC1) If $u: H_{0} \rightarrow G$ is a continuous homomorphism of a dense subgroup $H_{0}$ of a topological group $H$ into a Raŭkov-complete topological group $G$, then $u$ (uniquely) extends to a continuous homomorphism from $H$ into $G$. 
(RC2) The homeomorphism group (equipped with the compact-open topology) of a compact Hausdorff space is Raŭkov-complete.

Now assume $H_{0}$ is a dense subgroup of a weakly right amenable topological group $H$. Let $K \times H_{0} \ni(x, h) \mapsto x . h \in K$ be a (jointly) continuous affine right action of $H_{0}$ on a non-empty compact convex set $K$. For any $h \in H_{0}$ denote by $f_{h}: K \rightarrow K$ the homeomorphism $x \mapsto x \cdot h^{-1}$. It is clear that $\Phi_{0}: h \mapsto f_{h}$ is a homomorphism of $H_{0}$ into the homeomorphism group $G$ of $K$. What is more, the joint continuity of the action is equivalent to the continuity of this homomorphism. So, (RC1) combined with (RC2) implies that $\Phi_{0}$ extends to a continuous homomorphism $\Phi: H \rightarrow G$. We conclude that the given action (of $H_{0}$ on $K$ ) extends to a jointly continuous right action $(x, h) \mapsto \Phi\left(h^{-1}\right)(x)$ of $H$ on $K$. It is easy to verify that this action is affine and hence it has a fixed point, which finishes the proof.

\section{REFERENCES}

[1] E. Akhiezer, Recurrence in Topological Dynamics. Furstenberg Families and Ellis Actions, Springer Science+Business Media, New York, 1997.

[2] A.V. Arhangel'skii and M.G. Tkachenko, Topological Groups and Related Structures, Atlantis Press, Paris; World Scientific, Hackensack, NJ, 2008.

[3] E.W. Cheney, P.D. Morris, On the existence and characterization of minimal projections, J. Reine Angew. Math. 270 (1974), 61-76.

[4] M.M. Day, Fixed point theorems for compact convex sets, Illinois J. Math. 5 (1961), 585-590.

[5] M.M. Day, Correction to my paper "Fixed point theorems for compact convex sets", Illinois J. Math. 8 (1964), 713.

[6] I. Farah and S. Solecki, Extreme amenability of $L_{0}$, a Ramsey theorem, and Lévy groups, J. Funct. Anal. 255 (2008), 471-493.

[7] F.P. Greenleaf, Invariant Means on Topological Groups and Their Applications, Van Nostrand, New York, 1969.

[8] M. Gromov and M.D. Milman, A topological application of the isoperimetric inequality, Amer. J. Math. 105 (1983), 843-854.

[9] M. de Jeu, R. El Harti and P.R. Pinto, Amenable crossed product Banach algebras associated with a class of $C^{*}$-dynamical systems, Integral Equations Operator Theory 87 (2017), 169178.

[10] N. Dunford and J.T. Schwartz, Linear Operators. Part I: General Theory, Interscience Publishers, New York, 1958.

[11] S. Kakutani, Two fixed-point theorems concerning bicompact convex sets, Proc. Imp. Acad. Tokyo 14 (1938), 242-245.

[12] A.S. Kechris, V.G. Pestov and S. Todorcevic, Fraïssé limits, Ramsey theory, and topological dynamics of automorphism groups, Geom. \& Funct. Anal. 15 (2005), 106-189.

[13] D. Koehler and P. Rosenthal, On isometries of normed linear spaces, Studia Math. 36 (1970), 213-216.

[14] H. Lin, Residually finite-dimensional and AF-embeddable $C^{*}$-algebras, Proc. Amer. Math. Soc. 129 (2001), 1689-1696.

[15] A. Markov, Quelques théoremes sur les ensembles abéliens, Dokl. Akad. Nauk SSSR 10 (1936), 311-314.

[16] J. Melleray and T. Tsankov, Generic representations of abelian groups and extreme amenability, Israel J. Math. 198 (2013), 129-167.

[17] A.L.T. Paterson, Amenability (Mathematical Surveys and Monographs, Vol. 29), American Mathematical Society, Providence, Rhode Island, 1988.

[18] J.-P. Pier, Amenable Locally Compact Groups, Wiley-Interscience, New York, 1984.

[19] C. Rosendal and S. Solecki, Automatic continuity of homomorphisms and fixed points on metric compacta, Israel J. Math. 162 (2007), 349-371.

[20] W. Rudin, Projections on invariant subspaces, Proc. Amer. Math. Soc. 13 (1962), 429-432.

[21] W. Rudin, Functional Analysis, McGraw-Hill, Inc., New York, 1991.

[22] V. Runde, Lectures on Amenability (Lecture Notes in Mathematics, vol. 1774), SpringerVerlag, Berlin, 2002.

[23] M. Sabok, Extreme amenability of abelian $L_{0}$ groups, J. Funct. Anal. 263 (2012), 2978-2992.

[24] B. Sz.-Nagy, On uniformly bounded linear transformations in Hilbert space, Acta Sci. Math. (Szeged) 11 (1947), 152-157. 
P. Niemiec, Instytut Matematyki, Wydzial Matematyki i Informatyki, Uniwersytet Jagielloński, ul. ŁoJasiewicza 6, 30-348 Kraków, Poland

E-mail address: piotr.niemiec@uj.edu.pl

P. Wójcik, Instytut Matematyki, Uniwersytet Pedagogiczny, ul. Podchorążych 2, 30-084 Kraków, Poland

E-mail address: pwojcik@up.krakow.pl 